\title{
Immunization Coverage and Medicaid Managed Care in New Mexico: A Multimethod Assessment
}

\author{
Michael A. Schillaci, $P b D^{1}$ \\ Howard Waitzkin, MD, $P b D^{2}$ \\ E. Ann Carson, MS $S^{2,3}$ \\ Cynthia M. Lopez, DrPH \\ Deborab A. Boebm, MA \\ Leslie A. Lopez, MA \\ Sheila F. Maboney, MPH, CNM² \\ 'Department of Social Sciences, University \\ of Toronto at Scarborough, Toronto, Ontario, \\ Canada
}

${ }^{2}$ Department of Family and Community Medicine, University of New Mexico, Albuquerque, NM

${ }^{3}$ Department of Anthropology, University of New Mexico, Albuquerque, NM

\begin{abstract}
BACKGROUND We wanted to examine the association between Medicaid managed care (MMC) and changing immunization coverage in New Mexico, a predominantly rural, poor, and multiethnic state.

METHODS As part of a multimethod assessment of MMC, we studied trends in quantitative data from the National Immunization Survey (NIS) using temporal plots, Fisher's exact test, and the Cochran-Armitage trend test. To help explain changes in immunization rates in relation to MMC, we analyzed qualitative data gathered through ethnographic observations at safety net institutions: income support (welfare) offices, community health centers, hospital emergency departments, private physicians' offices, mental health institutions, managed care organizations, and agencies of state government.
\end{abstract}

RESULTS Immunization coverage decreased significantly after implementation of MMC, from $80 \%$ in 1996 to $73 \%$ in 2001 for the 4:3:1 vaccination series (Fisher's exact test, $P=.031$ ). New Mexico dropped in rank among states from 30th for this vaccination series in 1996 to 50th in 2001. A significant decreasing trend (Cochran-Armitage $P=.025$ ) in coverage occurred between 1996 and 2001 . Findings from the ethnographic study revealed conditions that might have contributed to decreased immunization coverage: (1) reduced funding for immunizations at public health clinics, and difficulties in gaining access to MMC providers; (2) informal referrals from managed care organizations and contracting physicians to community health centers and state-run public health clinics; and (3) increased workloads and delays at community health centers, linked partly to these informal referrals for immunizations.

CONCLUSIONS Medicaid reform in New Mexico did not improve immunization coverage, which declined significantly to among the lowest in the nation. Reduced funding for public health clinics and informal referrals may have contributed to this decline. These observations show how unanticipated and adverse consequences can result from policy interventions in complex insurance systems.

Ann Fam Med 2004;2:13-21. DOI: 10.1370/afm.100.

\section{INTRODUCTION}

Conflicts of interest: none reported

\section{CORRESPONDING AUTHOR}

Howard Waitzkin, MD, PhD

Department of Family and Community

Medicine

1 University of New Mexico, MSC09 5060

2400 Tucker Avenue NE

Albuquerque, NM 87131-0001

waitzkin@unm.edu.

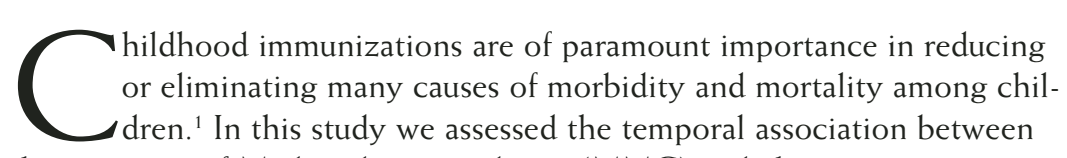
the initiation of Medicaid managed care (MMC) and changing immunization coverage in New Mexico, a predominantly rural, poor, and multiethnic state. We used a multimethod approach, incorporating quantitative sentinel events and qualitative ethnographic methodologies.

In July 1997 the state of New Mexico instituted mandatory managed care for Medicaid recipients. With a population of 1.8 million, New Mexico has ranked first in the percentage of its population lacking health insurance 
$(26 \%){ }^{2(p 102)}$ first in proportion of the population living in poverty $(21 \%),{ }^{2(p 444)}$ and sixth in the percentage of unemployed workers (4.9\%). ${ }^{2(\mathrm{p} 370)} \mathrm{MMC}$ has covered about $39 \%$ of the approximately 560,000 children in New Mexico. ${ }^{3}$ Transition to MMC occurred in both urban and rural counties.

The state government mandated that the 3 managed care organizations contracted under MMC provide immunizations to covered children. An underlying expectation was that MMC would improve the immunization rates. Partly because of this expectation, the state government reduced funding and personnel for immunization activities in the New Mexico Department of Health. These reductions also resulted from lower federal funding during 1997 for immunization infrastructure support. ${ }^{4}$

On the other hand, New Mexico and other states continued to receive funding from the Vaccines for Children program, initiated in 1994. As an entitlement program, Vaccines for Children has provided federally purchased immunizations for children who enrolled in Medicaid as well as for uninsured or underinsured children. ${ }^{4}$ New Mexico continued to fold Vaccines for Children funding into a "universal purchase" program, which provided vaccines to public clinics and participating private providers, including managed care organizations.

Although previous studies have assessed the impact of targeted interventions to improve immunization practices within local or regional Medicaid programs, ${ }^{5-9}$ surveillance of changing immunization coverage levels linked to state-level policy changes has not occurred to a substantial degree. Concrete modifications in health care workers' behaviors concerning immunizations after the transition to MMC have received little attention. Our multimethod project aimed to address these gaps in research on immunizations.

\section{METHODS}

\section{Overview of the Study}

We used 3 methods to assess the impact of MMC reform at 3 levels of analysis. First, to study effects on individuals, we conducted a population-based survey focusing on low-income ZIP codes in urban and rural counties. Second, we used an ethnographic method to assess the impacts on safety net institutions. A third method traced preventable adverse sentinel events, such as immunization rates, at the population level. ${ }^{10-12}$

Although the survey and ethnographic components of the study started in January 1997, about 6 months before implementation of MMC, the sentinel events component began as planned about 2 years later. Publicly available databases containing population-level indicators became available after a delay during which various state and federal agencies compiled the data. As data on immunization rates became available, we recognized that a major deterioration was taking place, which we had not expected. On the contrary, we had expected that immunization rates either would stay stable or would improve, because they were included as a mandated quality assurance indicator for the managed care organizations that participated in MMC.

As a result of the unexpected deterioration in immunization rates, we reexamined our ethnographic files and reviewed this problem with the ethnographic field researchers. Although we had not planned to focus on immunizations in the ethnographic component, we tried to use our previous ethnographic observations to clarify mechanisms by which MMC might have been associated with changing immunization practices. We also tried to redirect the ethnographic activities, so that observations during the remaining 2 years of the study could continue to track immunization practices.

\section{Methods in the Sentinel Events Component}

\section{Sources of Data}

The National Immunization Survey (NIS) collects information on immunizations using random household telephone interviews and by surveying vaccination providers. Although the NIS does not oversample from low-income or minority groups and does not include in-person interviews to compensate for households without telephones (at the time of our study, approximately $12 \%$ of New Mexico households lacked telephones ${ }^{13}$ ), weighting procedures adjust for these issues. ${ }^{14}$ Although state immunization registries eventually might prove more effective, ${ }^{15-18}$ the NIS remains the cornerstone of the US immunization surveillance program. ${ }^{19}$ A primary goal of the NIS is to provide estimates of immunization coverage for all 50 states, the District of Columbia, and 27 large urban areas. ${ }^{14}$ The NIS allows comparisons among states over time to help evaluate immunization programs. ${ }^{14}$ The NIS does not report data by insurance categories.

Public health officials typically use vaccination series for monitoring immunization coverage. The 4:3:1 series consists of 4 doses of diphtheria, tetanus, and acellular pertussis vaccines; 3 doses of poliovirus vaccine; and 1 dose of measles-containing vaccine, whereas the $4: 3: 1: 3$ series includes the previous vaccines plus 3 doses of Haemophilus influenzae type b vaccine. ${ }^{4,5}$ We chose the $4: 3: 1$ and 4:3:1:3 series rather than individual vaccines, such as hepatitis $B$, because these series provide useful measures of overall immunization levels in the population. NIS data included annual sample sizes, point estimates of immunization 
coverage levels, and confidence intervals for the point estimates. ${ }^{20-25}$ The NIS provided data on immunization coverage by provider type. ${ }^{14,26}$ Because the $4: 3: 1$ and 4:3:1:3 series showed the same trends, we report only the $4: 3: 1$ series here.

\section{Analysis}

Changes in immunization coverage levels were assessed by temporal plots of the data and by comparisons of coverage before and after MMC implementation. We used Fisher's exact test to assess the significance of changes in coverage levels ${ }^{27}$; a 1 -sided significance level of .05 was required for comparisons because we were aware that coverage levels had decreased ${ }^{28}$ (2-sided significance levels would be approximately twice as large as those reported here and would not change the major findings). We assessed trends in immunization coverage by the Cochran-Armitage trend test ${ }^{29,30}$ (SAS version 8.0 .2 , 2001). Post hoc power analyses, based on the NIS sample sizes, were conducted for all nonsignificant comparisons using the NCSS statistical software package (Number Cruncher Statistical Systems, 2002); we report the $\beta$, or probability of type II error, of these comparisons. SYSTAT software (SPSS Inc, 2001) was used to generate graphs.

\section{Methods in the Ethnographic Component}

\section{Rationale}

We have described our ethnographic methods in a previous report. ${ }^{31}$ In brief, ethnography refers to the systematic and in-depth description of cultural and social processes. $^{32}$ In health services research, ethnography can examine effects of policy changes on institutions and communities. ${ }^{33}$ The ethnographers' immersion in "local categories, local narratives, and local practices" 34 can promote in-depth understanding of patients' and providers' perspectives about MMC. In our research, a senior anthropologist supervised postdoctoral fellows and advanced graduate students.

\section{In-depth Interviews and Field Observations}

The ethnographic sampling procedures aimed to elicit experiences with MMC at the organizations most affected by the reform. Research assistants conducted interviews at income support (welfare) offices, community health centers, hospital emergency departments, private physicians' offices, and mental health institutions. Participant observation also took place at community meetings and public forums focusing on MMC. On-site participation involved 2, 30-hour periods of contact at the start of MMC and again 9, 18, and 27 months later at each of 11 health care sites in both urban and rural areas. Ethnographers conducted 15 to
20 interviews at each site in each of the 3 phases. They interviewed 39 clerical staff workers; 14 administrators; 32 nurses, nurse practitioners, physician's assistants, and medical assistants; 19 physicians; and 46 patients. In addition, senior members of the research group conducted structured interviews with the chief executive officers of the 3 managed care organizations participating in $\mathrm{MMC}$, as well as the directors of the state agencies that administered the program.

The interviews followed a standard protocol. A manager was interviewed first, and then follow-up interviews were conducted with staff, professionals, and clients. Four interview protocols were tailored for executives, administrators and professional staff, clerical staff, and patients. The interview protocols asked respondents to describe their experiences, both positive and negative, with MMC. Items within the protocols also asked respondents to relate the concrete impact that MMC exerted on their organizations or (for patients) on their health care. In addition, researchers at each site observed interpersonal interactions, especially in intake and referral processes.

\section{Collection of Documents}

Documents were collected at each site. This approach provided information about how formal, written documents described organizational procedures, how public and private organizations conceptualized the care they provided, and how organizational procedures differed before and after the transition to MMC. Information sent by the MMC program also was collected.

\section{Analysis}

The ethnographic work provided 3 sources of data: documents collected from sites, field notes taken by observers, and interview notes. Data were analyzed through a series of iterative readings or codings. Codes included such issues as "formularies," "primary care practitioner (PCP) assignment," "referrals," and "immunization," as well as safety net providers' and administrators' concerns, such as workload, financial impact, and continuity of care. From more than 100 codes, frequently occurring codes emerged as key themes. Through joint discussions, the ethnographic team then developed a coded index of topics addressed in the documents, field notes, and interview notes. ${ }^{35}$ This iterative group process aimed to reduce the impact of bias among individual researchers or the senior investigators who initiated the study. To facilitate data analysis, we used the ATLAS.ti software package. ${ }^{36}$

Analyses that emerged from this approach were integrated through triangulation of data, ie, by comparing documents, observations, and interviews, to develop a coherent and consistent description of repeated 
Table 1. National Immunization Survey Estimates of Immunization Rates, 4:3:1 Series, 95\% Confidence Intervals (Cl), and National Ranks for New Mexico from 1994 to 2001

\begin{tabular}{lllll}
\hline Year & Number* & $\begin{array}{l}\text { Percent } \\
\text { Coverage }^{\dagger}\end{array}$ & $\mathbf{9 5 \% ~ C l}$ & Rank $^{\S}$ \\
\hline 1994 & 326 & 73 & $65.6-80.4$ & 30 \\
1995 & 204 & 77 & $71.5-82.5$ & 25 \\
1996 & 270 & 80 & $75.3-84.7$ & 30 \\
1997 & 293 & 77 & $72.4-81.6$ & 31 \\
1998 & 254 & 73 & $66.7-79.3$ & 51 \\
1999 & 298 & 76 & $70.1-81.9$ & 43 \\
2000 & 309 & 72 & $66.3-77.9$ & 50 \\
2001 & 338 & 73 & $68.0-78.0$ & 50 \\
\hline
\end{tabular}

* Sample sizes for New Mexico provided by the National Immunization Survey. Appendix 1 reports the estimated proportion, during each year, of children immunized after the initiation of Medicaid managed care.

† National Immunization Survey for all 50 states and the District of Columbia.

\# $\mathrm{Cl}$ denotes confidence interval provided by the National Immunization Survey.

$\S$ Rank for immunization rates among states. For ties, average ranks were calculated by assigning duplicate values (ties) consecutive ranks and dividing the sum of those consecutive ranks by the number of duplicate values. Data for 1994-1995 apply to all 50 states and for 1996-2001 apply to all 50 states and the District of Columbia.

themes, concerns, and meanings. ${ }^{37,38}$ This process led to thematic categories, each with examples or illustrative quotations from respondents. In later meetings of the research group, we selected quotations that best captured the thematic categories. We report here those themes and examples pertinent to immunization.

\section{RESULTS}

\section{Trends in State and National Immunization Rates}

After the initiation of MMC in July 1997, immunization coverage levels fell to among the lowest in the nation. In 1996 immunization rates for New Mexico ranked 30th for the 4:3:1 vaccination series. By 1998 New Mexico slipped in rank to last among the 50 states and the District of Columbia and then increased slightly in 1999 to 43rd and 46th. In 2000 and 2001, however, New Mexico ranked 50th (Table 1).

Temporal trends indicated a marked decrease in immunization coverage coincident with the implementation of MMC (Table 1 and Figure 1). This pattern contrasted with national trends indicating stability or an increase between 1996 and 1998. The decrease for New Mexico between 1996 and 1998 was significant (Fisher's exact test, $P=.034$ ). Although immunization coverage in 1999 remained lower than in 1996, the difference was not significant (Fisher's exact test, $P=.226, \beta=.854$ ). Comparison of the 1996 immunization coverage level with that reported for 2001, however, was significant (Fisher's exact test, $P=.031)$. The small rebounds during 1999 and 2001 did not represent statistically significant changes. Results from the Cochran-Armitage test assessing temporal trends indicated a significant decreasing trend between 1996 and $2001(P=.025)$.
We further analyzed the data to account for the time lag that resulted from the method NIS used to elicit data. This analysis, presented in Appendix 1, determined that in the 1997 NIS data, approximately $26 \%$ of the immunizations occurred after the implementation of MMC in July 1997. In the 1998 NIS data, approximately $78 \%$ of the immunizations occurred after MMC implementation. For 1999 and later years, all immunizations took place after initiation of MMC.

The trends in immunization coverage by provider type showed a decrease for public providers and an increase for private providers between 1997 and 1998

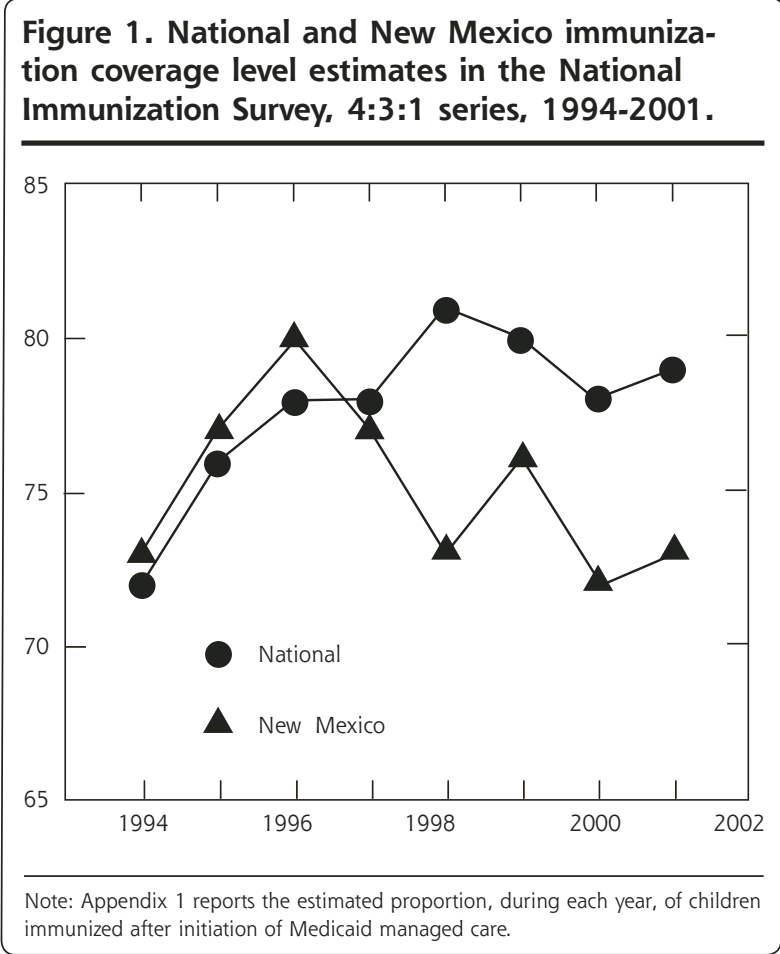




\section{Figure 2. New Mexico immunization coverage level estimates by provider type, National Immu- nization Survey, 4:3:1 series, 1996-2000.}

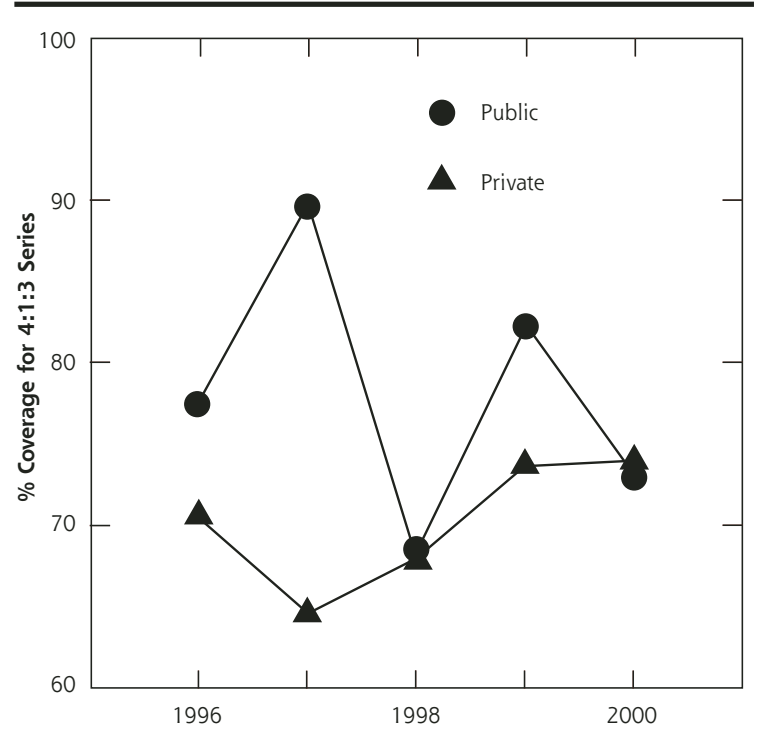

Note: Public providers include public health clinics, public hospitals, and community health centers; private providers include private clinics, group practices, and managed care organizations. Data on provider type were not reported in the NIS before 1996; data for 2001 were not available for New Mexico because of insufficient sample size.

(Figure 2). Coverage by public providers was higher than for private providers before MMC implementation, whereas equal levels of coverage by these two provider types were observed after the reform. The decrease in immunization coverage for public providers between 1996 and 1998 was not significant (Fisher's exact test, $P=.139 ; \beta=.788$ ). Although immunizations by public providers increased somewhat in 1999, they declined again in 2000. Meanwhile, immunizations by private providers increased slightly in 1999 and 2000, but to only $2 \%$ higher than in 1996 .

\section{Ethnographic Findings}

\section{General Findings}

At clinical sites, we observed major increases in workload, attributable to new managerial requirements. ${ }^{31}$ Paperwork increased markedly, because of changed eligibility and referral procedures. Lack of training heightened the perceived impact. In both safety net institutions and managed care organizations, staff turnover and frequently changing policies impeded development of adequate knowledge to cope with administrative demands.

Financial Stress Among Safety Net Providers Respondents at most clinical sites reported reduced income. ${ }^{31}$ This change resulted from delays in contracting with providers, denials for claims of patients who sought care at customary sites instead of the newly assigned primary care providers, and other denied claims. The MMC program randomly assigned many patients to primary care practitioners, rather than to their previous safety net providers. As a result, these safety net practitioners lost capitation payments for patients whom they previously had attended. Payments from managed care organizations for capitated Medicaid patients were delayed, resulting in substantial cash-flow problems.

\section{Findings Pertinent to Immunizations}

In this context, the ethnographic findings clarified processes that might have contributed to declining immunizations: (1) reduced funding for immunizations at public health clinics and difficulties in gaining access to MMC clinicians; (2) informal referrals for immunizations from facilities that served Medicaid patients, including managed care organizations and offices of contracting physicians, to community health centers and state-run public health clinics; and (3) increased workloads and delays at community health centers, linked partly to these informal referrals for immunizations. When we interviewed officers of managed care organizations and state government agencies (about 3 years after MMC initiation), they did not express awareness of changed immunization rates or practices.

\section{Reduced Funding and Access Difficulties}

Reduced funding for immunizations at public health clinics and difficulties in gaining access to MMC clinicians affected delivery of vaccinations. For example, a public health clinic nurse manager commented:

...in the last year [to] 15 months, we've had about $50 \%$ of our budget cut, and what that means in practice is that we're down about $50 \%$ of our nursing staff here in [the] county. So, if someone wants immunizations, instead of taking walk-ins, we're only doing appointments. Sometimes we can squeeze them in during the same week, but they're maybe waiting 2 to 3 weeks for an appointment. This is also a big problem for people who can't get in to see their Medicaid provider.

Such problems of access might have contributed to the falling immunization rates.

\section{Informal Referrals}

In addition, some facilities that served Medicaid patients informally referred patients to community health centers and state-run public health clinics to receive immunizations, even though the patient's primary care practitioner was located at the facility 
referring the patient. As one community health clinic pediatrician described:

Parents tell me that they have gone over to the Del Norte [a pseudonym for 1 of 3 New Mexico managed care organizations] building because their kids need shots to stay in school, and they say that they [Del Norte] can't see them for 1 or 2 months. Parents will usually say "[Del Norte] told us to come here." So they come here. If Del Norte is here for primary care - and I know they are busy too-I think they should have some way to take care of this kind of thing. [The families] are there- they shouldn't be sent away. If you have the opportunity to get kids caught up on their shots, there should be some way to make that available.

Some Medicaid recipients seeking immunizations, who could not make a timely appointment to see their primary care practitioners, had to seek care at safety net providers.

\section{Workloads and Delays}

Respondents referred to the cost, inconvenience, and time constraints of providing immunizations at managed care organizations as a motivation for these referrals to safety net institutions. When asked why primary care practitioners from the managed care organizations were sending their patients to local community health centers for immunizations, a medical assistant working at a center serving primarily urban-dwelling American Indians responded:

It's because immunizations are time consuming. There are 3 pieces of paper for each immunization. You have to record the lot number too. It's very time consuming. You really wear out your personnel doing immunizations. I think that's why they [primary care physicians at managed care organizations] send their Medicaid clients to us for immunizations.

A nurse practitioner at the same clinic stated:

When I see a child for immunizations who is not on Medicaid, I can't do a physical. I can do a physical on kids who have Medicaid because Medicaid will pay for a physical with immunizations. In fact, Medicaid requires a physical with immunizations. I get angry because if this child has a primary care physician but the PCP won't do immunizations, they send the child to us. If the child has Medicaid, then I have to do a physical as required by Medicaid, which repeats the work. The EPDST [Early Prevention Developmental Screening Test] form also has to be filled out, but this only has to be filled out for people who are on [Medicaid]. This form is time consuming, so more time is spent on the form with Medicaid patients.

The ethnographic interviews therefore suggested that informal referrals for immunizations by managed care organizations and private physicians might have led to increased workloads and delays at community health centers.

\section{DISCUSSION}

\section{Unanticipated Consequences of Policy Change in Complex Systems}

In New Mexico, immunization coverage did not increase after MMC. This finding is consistent with research showing that $\mathrm{MMC}$ has not consistently improved immunizations when compared with fee-for-service Medicaid. ${ }^{7-9}$ Declines in national rank for New Mexico paralleled declines in immunizations delivered by public providers. Based on the CDC estimate of approximately 40,000 New Mexico children eligible for immunization (NIS, unpublished data, 2003), a reduction in immunization rate of $8 \%$ to $10 \%$ translated into 3,000 to 4,000 additional children who did not receive immunizations in a given year. Estimates for 2002 indicated that New Mexico ranked next to the lowest among states in immunization rates. ${ }^{39}$

In contrast, several studies have assessed the results of focused interventions to improve immunization practices. An intervention that initiated a community-wide reminder, recall, and outreach system has succeeded in reducing geographical, racial, and ethnic disparities in immunizations. ${ }^{6}$ Within a specific MMC program, focused outreach efforts to reach underimmunized children also have improved immunization rates. ${ }^{5}$ Local studies (as opposed to our statewide research) have shown that MMC has sometimes but not always improved immunization coverage when compared with fee-for-service Medicaid..$^{7-9}$ Analysis of national NIS data has shown that timely immunization remains suboptimal in the United States. ${ }^{40}$

Because of unanticipated consequences, some analysts have questioned managed care in rural states. ${ }^{41,42}$ Specifically, Medicaid revenues for safety net institutions have tended to decrease under MMC, compromising the ability of these institutions to care for rural Medicaid and uninsured patients. ${ }^{43}$ In primary care practices, unanticipated consequences can follow interventions intended to improve quality and to reduce practice variations. ${ }^{44}$ Complexity theory helps explain these consequences in complex systems, where interventions might not lead to predictable outcomes. ${ }^{45-47} \mathrm{~A}$ similar unpredictability arises in other organizational settings, including such insurance programs as MMC. 
Regarding causality, we could not determine whether MMC directly caused the changes in immunization coverage. Our ethnographic data suggest that these unexpected outcomes might have resulted from complex, system-level changes. From the standpoint of complexity theory, unintended consequences can result from the nonlinear and unpredictable relationships between policy changes and the responses of complex health systems. ${ }^{46,47}$ Budget cuts for state public health clinics and difficulties in access to MMC clinicians might have contributed to lower immunization coverage. Reduced capacity for walk-in visits at public health clinics, combined with waiting times for appointments at managed care organizations, has increased barriers to immunizations. Similar barriers have led to inadequate immunizations in other states. ${ }^{48-50}$ Although such qualitative research cannot establish causality, the ethnographic observations clarified processes that plausibly contributed to declining immunization rates.

Informal referrals to public health clinics and community health centers by managed care organizations and private contracting physicians have exacerbated these barriers by increasing workload for safety net institutions. In our ethnographic observations, for instance, we observed this phenomenon at an urban clinic serving American Indians. A later phase of our research, not reported here, has found similar changes at Indian Health Service hospitals and clinics. Such phenomena are not unique to New Mexico. ${ }^{49}$ In addition to time and labor costs, clinicians listed inadequate Medicaid reimbursement as the primary reason for referrals to local health departments for immunizations. ${ }^{49,51-53}$ Although some states with MMC have shown comparatively high immunization coverage, our research suggests that practical disincentives for immunizations at managed care organizations and concomitant reduction in funding for public health clinics and community health centers may have contributed to the decline of immunizations.

\section{Alternative Explanations and Limitations of the Study}

Because the NIS asks about immunization experiences during the previous 2 years, the decline in immunization coverage noted in 1998 might have reflected changed practices that slightly antedated New Mexico's implementation of MMC in mid 1997. In addition, initial assignment of primary care clinicians under MMC might have disrupted established physician-patient relationships, leading to reduced immunizations. These explanations, however, do not account for the continued decline in coverage over several years.

Despite the Vaccines for Children program, organi- zational and budgetary changes at the Department of Health, partly a result of cutbacks in federal funding, ${ }^{4}$ might have reduced vaccine distribution and monitoring. Because New Mexico does not maintain accessible data on immunization doses delivered under the Vaccines for Children program, we could not evaluate changes specifically related to this program. An alternative explanation involving the Vaccines for Children program, however, cannot account for the continued decline in coverage and the informal referrals for immunizations_often termed "dumping" — reported by respondents at community health centers. In contrast with New Mexico, improved financing for immunizations by primary care practitioners under the Vaccines for Children program in some states led to reduced referrals to health departments. ${ }^{15,53}$

Declines in New Mexico's immunization coverage could have reflected changing practices that involved children outside the Medicaid system, especially the uninsured, rather than those affected directly by Medicaid reform. Because the NIS does not ask about respondents' insurance, we cannot exclude this possibility. On the other hand, any unfavorable changes affecting the uninsured might have reflected secondary effects of MMC implementation, as stress increased for safety net institutions providing immunizations for uninsured children; such secondary effects emerged as a major finding from our broader study of MMC in New Mexico. ${ }^{31,41}$ These secondary effects show that unexpected consequences can follow policy changes in complex systems. ${ }^{45,46}$

Statistical uncertainty also tempers our conclusions. As shown in the fairly wide confidence intervals around the annual point estimates of immunization coverage (Table 1), this uncertainty derives partly from the small NIS sample sizes at the state level. NIS sample sizes from 1994 to 2001 in New Mexico ranged from 204 to 338 respondents. In addition, New Mexico has ranked first or second in the nation for households without telephone service. ${ }^{13}$ Despite statistical adjustment for this potential source of bias, ${ }^{14}$ the true coverage levels for the low-income population in New Mexico might be worse than those estimated by the NIS.

\section{CONCLUSIONS}

In summary, immunization rates in New Mexico fell after 1996 to among the lowest in the United States. Decreasing immunization rates after MMC implementation indicate that this reform did not improve immunization coverage. Although we could not fully determine the causal impact of $\mathrm{MMC}$, our ethnographic observations indicated that MMC played a plausible role in initiating complex systems-level changes associated with declining immuni- 
zations. Specifically, the ethnographic findings suggested that a reduction in funding to state-run public health clinics, in conjunction with increased informal referrals by private physicians and managed care organizations, might have contributed to decreased immunization coverage. Future policy decisions should consider the direct and indirect effects of Medicaid reform on safety net institutions responsible for immunizations and other necessary preventive services.

\section{APPENDIX 1.}

Estimated Proportion of Children Immunized after the Initiation of Medicaid Managed Care

We further analyzed the data to account for the time lag that resulted from the method of eliciting data used by the NIS. Not all children 19 to 35 months of age who were included in the NIS report for any given year actually received their immunizations that year. For instance, children included in the 1997 NIS report were born between February 1994 and May 1996; those included in the 1998 NIS report were born between February 1995 and May 1997. Although the American Academy of Pediatrics recommends that childhood immunizations be completed by 18 months of age, parents typically do not bring their children to a health care provider to receive the last series of childhood immunization until the customary 2-year visit. As a result, most children included in the 1997 NIS report received their last childhood immunizations between February 1996 and May 1998, 24 months after birth. The NIS did not include in their 1997 survey any children immunized after December 1997. For 1997, the immunization rates reported by the NIS therefore reflected immunizations that occurred over the 23-month period between February 1996 and December 1997.

If it is assumed that the distribution of immunizations is uniform across months, approximately $26 \%$ (ie, 6 of 23 months) of the immunizations reported for 1997 occurred after the implementation of MMC in July 1997. Similarly, the children included in the 1998 NIS report likely received their immunizations between February 1997 and December 1998. Again, assuming the distribution of immunizations is even across months, approximately $78 \%$ (ie, 18 of 23 months) of the immunizations occurred after the implementation of MMC in July 1997. By 1999 all immunizations occurred after the implementation of MMC.

To read commentaries or to post a response to this article, see it online at http://www.annfammed.org/cgi/content/full/2/1/13.

Key words: Immunizations/in infancy and childhood; vaccination; Medicaid; managed care programs

Submitted May 5, 2003; submitted, revised, September 3, 2003; accepted October 12, 2003.

Funding support: This research was supported in part by grants from the Agency for Healthcare Research and Quality (R01 HS09703), the National Institute of Mental Health (1R24 MH58404 and 1R25 MH60288), the New Mexico Department of Health (98/665.42.194), the World Health Organization, the Hospital of the University of New Mexico, and the Dedicated Health Research Funds of the University of New Mexico School of Medicine. The views expressed in this article do not necessarily reflect those of the funding agencies.

Acknowledgments: We thank Dr. Gary Simpson of the New Mexico Department of Health and Drs. Louise Lamphere, Richard Santos, William Wiese, Robert Williams, and Cathleen Willging of the University of New Mexico for their advice, comments, and criticisms.

\section{References}

1. Impact of vaccines universally recommended for children - United States, 1990-1998. MMMR Morb Mortal Wkly Rep. 1999;48:243-248.

2. U.S. Census Bureau. Statistical abstract of the United States: 2001 Washington, DC: Government Printing Office; 2001. Available at: http://www.census.gov/prod/2002pubs/01statab/stat-ab01.html. Accessed December 1, 2002.

3. Profile of general demographic characteristics. Available at: http:// www.edd.state.nm.us/FACTBOOK/WORKFORCE/NMProfile.pdf. Accessed September 1, 2003. New Mexico Human Services Department. Monthly all children eligibility report. Available at: http:// www.state.nm.us/hsd/mad/pdf_files/Reports/AllChildDistributionby Co.pdf. Accessed September 1, 2003.

4. Freed GL, Clark SJ, Cowan AE. State-level perspectives on immunization policies, practices, and program financing in the 1990s. Am J Prev Med. 2000;19:32-44.

5. Vivier PM, Alario AJ, O'Haire C, Dansereau LM, Jakum EB, Peter G. The impact of outreach efforts in reaching underimmunized children in a Medicaid managed care practice. Arch Ped Adol Med. 2000;154: 1243-1247.

6. Szilagyi PG, Schaffer S, Shone L, et al. Reducing geographic, racial, and ethnic disparities in childhood immunization rates by using reminder/recall interventions in urban primary care practices. Pediatrics. 2002; 110:e58.

7. Alessandrini EA, Shaw KN, Bilker WB, Schwartz DF, Bell LM. Effects of Medicaid managed care on quality: childhood immunizations. Pediatrics. 2001;107:1335-1342.

8. Szilagyi PG. Medicaid managed care and childhood immunization delivery. J Public Health Manage Pract. 1998;4:67-72.

9. Wood D, Halfon N, Sherbourne C, Grabowsky M. Access to infant immunizations for poor, inner-city families: what is the impact of managed care? J Health Care Poor Underserved. 1994;5:112-123.

10. Rutstein DD. The principle of the sentinel health event and its application to occupational diseases. Arch Env Health. 1984;39:158.

11. Rutstein DD, Berenberg W, Chalmers TC, Child CG, Fishman AP, Perrin EB. Measuring the quality of medical care: a clinical method. N Engl J Med. 1976;294:582-588.

12. Rutstein DD, Mullan RJ, Frazier JM, Halperin WE, Melius JM, Sesito JP. Sentinel health events (occupational): a basis for physician recognition and health surveillance. Am J Public Health. 1983;73:1054-1062.

13. U.S. Census Bureau. Historical census of housing tables: telephones. Available at: http://www.census.gov/hhes/www/housing/census/ historic/phone.html. Accessed September 1, 2003.

14. Zell ER, Ezzati-Rice TM, Battaglia MP, Write RA. National Immunization Survey: the methodology of a vaccination surveillance system. Public Health Rep. 2000;115:65-77.

15. The National Vaccine Advisory Committee. Strategies to sustain success in childhood immunizations. JAMA. 1999;282:363-370.

16. Progress in development of immunization registries - United States, 2000. MMMR Morb Mortal Wkly Rep. 2001;50:3-7.

17. Cordero JF, Orenstein WA. The future of immunization registries. Am J Prev Med. 1997;S122-S124.

18. Horlick GA, Beeler SF, Linkins RW. A review of state legislation related to immunization registries. Am J Prev Med. 2001;20:208-213.

19. Fairbrother G, Freed GL, Thompson JW. Measuring immunization coverage. Am J Prev Med. 2000; 19:78-88.

20. State and national vaccination coverage levels among children aged 19-35 months - United States, April-December 1994. MMWR Morb Mortal Wkly Rep. 1995;44:613,619-623.

21. National, state, and urban area vaccination coverage levels among children aged 19-35 months - United States, January-December 1995. MMWR Morb Mortal Wkly Rep. 1997;46:176-182. 
22. Status report on the Childhood Immunization Initiative: national, state, and urban area vaccination coverage levels among children aged 19-35 months - United States. 1996. MMW/R Morb Mortal W/kly Rep. 1997;46:657-664.

23. National, state, and urban area vaccination coverage levels among children aged 19-35 months - United States. 1997. MMWR Morb Mortal Wkly Rep. 1998;47:547-554.

24. Herrera GA, Smith P, Daniels D, et al. National, state, and urban area vaccination coverage levels among children aged 19-35 months - United States. 1998. MMWR Morb Mortal Wkly Rep. 2000;49:1-26.

25. National, state, and urban area vaccination coverage levels among children aged 19-35 months - United States. 2000. MMWR Morb Mortal Wkly Rep. 2001;50:637-641.

26. Smith PJ, Bataglia MP, Huggins VJ, et al. Overview of the sampling design and statistical methods used in the National Immunization Survey. Am J Prev Med. 2001;20:17-24.

27. Fisher RA. The Design of Experiments. Edinburgh: Oliver and Boyd; 1935.

28. Selvin S. Statistical Analysis of Epidemiological Data. New York, NY: Oxford University Press; 1991:467.

29. Cochran WB. Some methods of strengthening the common $X^{2}$ tests. Biometrics. 1954; 10:417-451.

30. Armitage $P$. Tests for linear trends in proportions and frequencies. Biometrics. 1955:11:375-386.

31. Waitzkin H, Williams RL, Bock JA, McCloskey J, Willging C, Wagner W. Safety-net institutions buffer the impact of Medicaid managed care: a multi-method assessment in a rural state. Am J Public Health. 2002;92:598-610

32. Hahn R, ed. Anthropology in Public Health: Bridging Differences in Culture and Society. New York, NY: Oxford University Press; 1999.

33. Okongwu AF, Mencher JP. The anthropology of public policy: shifting terrains. Ann Rev Anthropol. 2000;29:107-124.

34. Kleinman A. Moral experience and ethical reflection: can ethnography reconcile them? A quandary for the "the new bioethics." Daedalus. 1999;138:69-97.

35. Emerson RM, Fretz RI, Shaw LL. Writing Ethnographic Fieldnotes. Chicago, Ill: University of Chicago Press; 1996.

36. Muhr T. Atlas.ti, version 4.2. Berlin, Germany: Scientific Software Development; 1999

37. Huberman AM, Miles MB. Data management and analysis methods. In: Denzin NK, Lincoln YS, eds. Handbook of Qualitative Research. Thousand Oaks, Calif: Sage; 1994.
38. Boyatzis RE. Transforming Qualitative Information. Thousand Oaks, Calif: Sage Publications; 1998.

39. Centers for Disease Control and Prevention. Immunization coverage in the U.S. Available at: http://www.cdc.gov/nip/coverage/NIS/02/toc02.htm. Accessed September 1, 2003.

40. Luman ET, McCauley MM, Stokley S, Chu SY, Pickering LK. Timeliness of childhood immunizations. Pediatrics. 2002;110:935-939.

41. Kronick R, Goodman DC, Wennberg J, Wagner E. The market place in health care reform: the demographic limitations of managed competition. N Engl J Med. 1993;328:142-152.

42. Silberman P. Poley S. James K. Slifkin R. Tracking Medicaid managed care in rural communities: a fifty-state follow-up. Health Aff $2002 ; 21: 255-263$

43. Horton S, McCloskey J, Todd C, Henriksen M. Transforming the safety net: responses to Medicaid managed care in rural and urban New Mexico. Am Anthropol. 2001;103:733-746.

44. Crabtree BF, Miller WL, Stange KC. Understanding practice from the ground up. J Fam Pract. 2001;50:881-887.

45. Miller WL, Crabtree BF, McDaniel RR, Jr., Stange KC. Understanding change in primary care practice using complexity theory. J Fam Pract. 1998;46:369-376

46. Miller WL, McDaniel Jr RR, Crabtree BF, Stange KC. Practice jazz: understanding variation in family practices using complexity science. J Fam Pract. 2001;50:872-878

47. McDaniel Jr RR, Driebe DJ. Complexity science and health care management. Adv Strat Manage. 2001;2:11-36.

48. Physician vaccination referral practices and vaccines for children - New York, 1994. MMWR Morb Mortal W/kly Rep. 1995;44:3-6.

49. Mayer ML, Clark SJ, Konrad TR, Freeman VA, Slifkin RT. The role of state policies and programs in buffering the effects of poverty on children's immunization receipt. Am J Public Health. 1999;89:164-170.

50. Bordley WC, Freed GL, Garrett JM, Byrd CA, Meriwether R. Factors responsible for immunization referrals to health departments in North Carolina. Pediatrics. 1994;94:376-380.

51. Lannon C, Brack V, Stuart J, et al. What mothers say about why poor children fall behind on immunizations: a summary of focus groups in North Carolina. Arch Ped Adol Med. 1995;149:1070-1075.

52. Santoli JM, Barker LE, Lyons BH, Gandhi NB, Philips C, Rodewald LE. Health department clinics as pediatric immunization providers. Am J Prev Med. 2001;20:266-271.

53. Szilagyi PG, Humiston SG, Shone LP, Barth R, Kolasa MS, Rodewald LE. Impact of vaccine financing on vaccinations delivered by health department clinics. Am J Public Health. 2000;90:739-745. 